\title{
UNA NUEVA ESPECIE DE CYRTOCARPA (ANACARDIACEAE) DE MÉXICO
}

\author{
Rosa María Fonseca \\ Laboratorio de Plantas Vasculares, Departamento de Biología, \\ Facultad de Ciencias, Universidad Nacional Autónoma de México \\ 04510 México, D.F.
}

\section{RESUMEN}

Se describe como nueva y se ilustra Cyrtocarpa kruseana, de los estados mexicanos de Chiapas, Guerrero, Jalisco y Nayarit. La presente especie es similar, en lo que se refiere a la posición y tamaño de inflorescencias a Cyrtocarpa velutinifolia de América del Sur y es diferente de las especies mexicanas, especialmente en el tamaño y posición de las inflorescencias así como en el tamaño del fruto y la época de floración y fructificación.

Palabras clave: Anacardiaceae, Cyrtocarpa kruseana, México.

\begin{abstract}
Cyrtocarpa kruseana, from the Mexican states of Chiapas, Guerrero, Jalisco and Nayarit, is described as new and illustrated. This species is similar to Cyrtocarpa velutinifolia from South America on account of the inflorecense size and position, and it differs from the Mexican species especially in the size of the fruit, size and position of the inflorescence, and phenology.
\end{abstract}

Key words: Anacardiaceae, Cyrtocarpa kruseana, Mexico.

Durante la realización del estudio taxonómico de las Anacardiaceae de Guerrero, se localizaron ejemplares de herbario determinados como Pseudosmodingium, Spondias, Tapirira y Bursera, cuyos atributos no correspondían a ninguno de estos géneros. Al revisar sus características se determinó que pertenecían a la familia Anacardiaceae; se trata de una especie que puede ubicarse dentro la tribu Spondiadeae la cual, de acuerdo con Engler (1892), se caracteriza por presentar 5 o 4(3) carpelos unidos, ocasionalmente más o muy rara vez sólo 
uno; estilos generalmente libres; óvulo uno por lóculo, péndulo del ápice de la cavidad; estambres en 2 series; fruto multilocular, raramente 2 o 1 lóculo; semillas 5 a 3, 2 o 1 por aborto; embrión por lo general recto (excepto Tapirira); hojas pinnadas, rara vez trifolioladas.

Entre los 18 géneros que se consideran dentro de Spondiadeae (Barkley, 1957; Wannan y Quinn, 1991), esta especie pertenece a Cyrtocarpa, de acuerdo con la siguiente clave modificada de Mitchell y Daly (1991) para géneros americanos de Spondiadeae.

1. Hojas con venas intramarginales; endocarpo óseo encerrado en una matriz fibrosa; pétalos valvados Spondias

1. Hojas sin venas intramarginales; endocarpo cartilaginoso u óseo; matriz fibrosa ausente; pétalos imbricados o valvados.

2. Ovario unilocular; endocarpo sin opérculos; embrión curvo; hojas perennes, no agrupadas hacia el ápice de las ramillas; folíolos peciolulados Tapirira

2. Ovario con 1 a 6 lóculos; endocarpo con 1 a 6 opérculos; embrión recto; hojas deciduas, agrupadas hacia el ápice de las ramillas; folíolos sésiles o subsésiles.

3. Opérculos 5 o 6; semillas usualmente 5 o 6 ; pétalos valvados o imbricados Antrocaryon

3. Opérculos 1 a 5 ; semillas generalmente 1 a veces 2 ; pétalos imbricados Cyrtocarpa

En consecuencia se propone a continuación:

Cyrtocarpa kruseana Fonseca sp. nov. Fig. 1

Arbores dioeciae; truncus usque $18 \mathrm{~cm}$ diametro, cortice rubro laevi exfolianti; ramuli leviter succulenti, in siccitate rugati; ramuli, folia juvenia et inflorescentiae ferrugineo-pubescentes. Folia decidua plerumque imparipinnata, $29-32 \mathrm{~cm}$ longa, petiolis $6.5-10 \mathrm{~cm}$ longis, foliolis sessilibus vel subsessilibus (5)7-9(10) paribus. Inflorescentiae paniculatae axillares, 10-17(20) cm longae, ad ramorum longorum apices aggregatae. Flores pentameri albo-viriduli; stamina 10; styli et stigmata 5 ad ovarii tertiam parte superam affixi, ovarium 5-loculare, ovulis in quoque loculo solitariis pendulis. Fructus drupacei usque $1.2 \mathrm{~cm}$ longi; endocarpium 3-4(5) operculis inaequalibus, 3-4(5) loculis inaequalibus. Semen 1 in loculo majore situm.

Árboles dioicos, de 4 a 6(9) $\mathrm{m}$ de altura, tronco hasta de $18 \mathrm{~cm}$ de diámetro, corteza roja a café rojiza, exfoliante. Ramas de color café, glabras, estriadas, con abundantes lenticelas y cicatrices de las hojas bien marcadas. Últimas ramas rojizas, lisas y algo carnosas en fresco, en seco con algunas arrugas, esparcida a densamente 


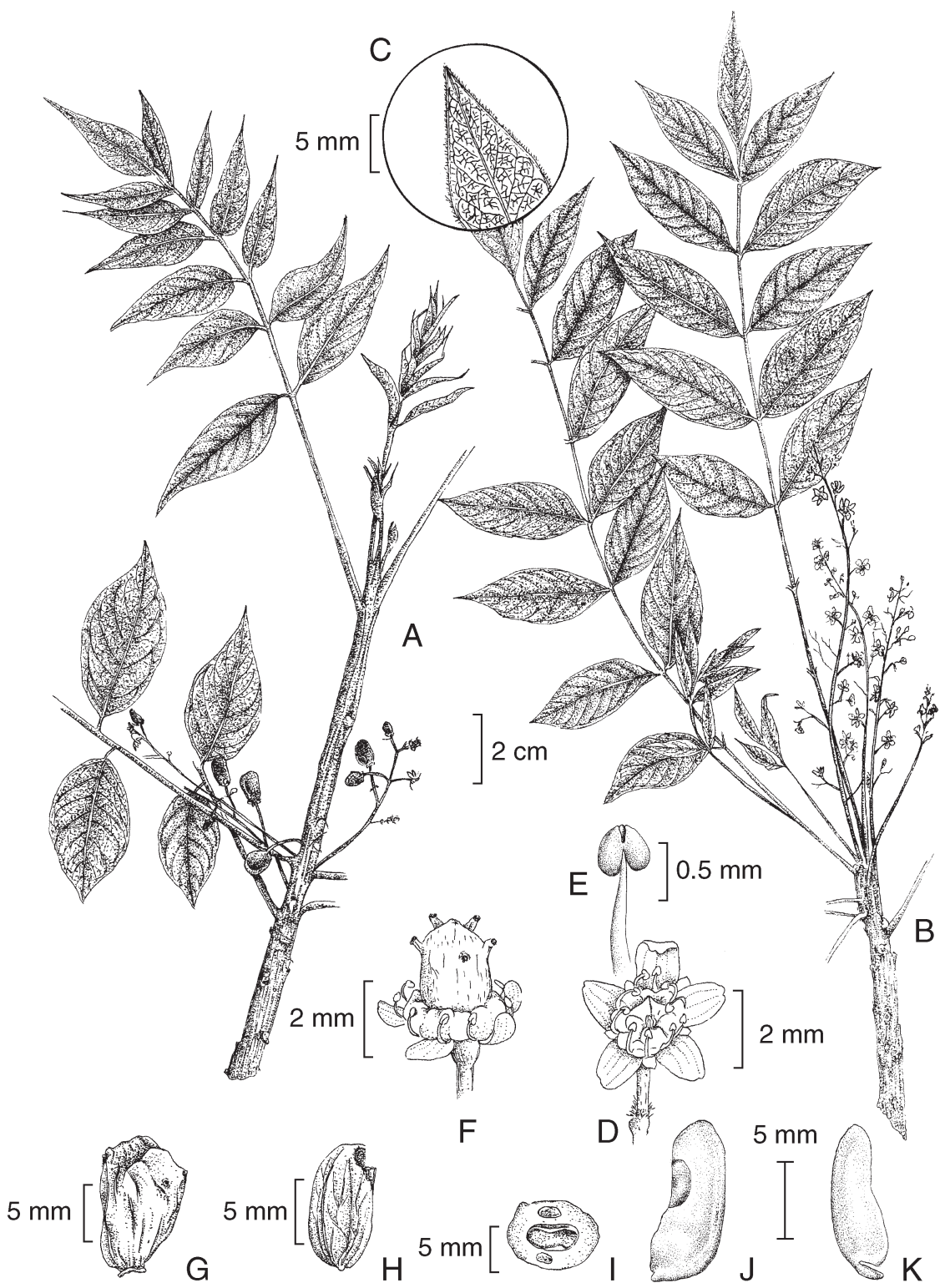

Fig. 1. Cyrtocarpa kruseana A. Planta con flores femeninas y frutos jóvenes; B. Rama con inflorescencias masculinas; C. Detalle de la venación; D. Flor masculina; E. Estambre; F. Flor femenina; G. Fruto; H. Endocarpo; I. Corte transversal del endocarpo mostrando tres lóculos y una semilla en el lóculo medio; J. Semilla; K. Embrión; Basado en A. Núñez 776 (A y F), H. Kruse 2847 (B, C, D y E) y H. Kruse 2878 (G, H, I, J y K). 
ferrugíneo-tomentosas, con tricomas rojos multicelulares, yemas axilares densamente ferrugíneo-tomentosas.

Hojas deciduas, agrupadas hacia el ápice de las últimas ramas, de hasta $32 \mathrm{~cm}$ de largo, pinnado compuestas, imparipinnadas, en ocasiones aparentemente paripinnadas, o uno de los folíolos del último par fusionado en la base con el folíolo terminal, verdes en el haz, ligeramente pálidas en el envés; pecíolos de 6.5 a 10 cm de largo; folíolos subopuestos, (5)7 a 9(10) pares; folíolos laterales sésiles o subsésiles, los inferiores con un pecíolulo de hasta $3 \mathrm{~mm}$ de largo, elípticolanceolados a elípticos, ligeramente inequiláteros, ápice agudo a acuminado, margen entero, base aguda a cuneada, de 4 a $9 \mathrm{~cm}$ de largo y de 2 a $3.5 \mathrm{~cm}$ de ancho; folíolo terminal sésil o peciolulado, con pecíolulo hasta de $1.5 \mathrm{~cm}$, lámina de $6 \mathrm{a}$ $8 \mathrm{~cm}$ de largo, por 1.9 a $2.5 \mathrm{~cm}$ de ancho, ápice agudo a acuminado, base largamente decurrente, todos membranosos, venación de color café claro a amarillenta en el haz, con 8 a 11 venas secundarias en cada mitad de la lámina, pubescentes en ambas superficies con tricomas blancos, erectos a ligeramente adpresos y tricomas rojos glandulares, cuando jóvenes densamente ferrugíneas.

Inflorescencias en panículas axilares, en las axilas de las hojas del mismo año, agrupadas hacia el ápice de las últimas ramas, de 10 a $17(20) \mathrm{cm}$ de largo, 1.5 a $2 \mathrm{~cm}$ de ancho; pedúnculo de 2 a $4 \mathrm{~cm}$ de largo, pubescente, raquis pubescente, con tricomas blancos y con tricomas rojos glandulares; ramas secundarias de la inflorescencia paniculiformes de hasta $1.2 \mathrm{~cm}$ de largo; brácteas triangulares de 0.5 a $0.6 \mathrm{~mm}$ de largo, densamente ferrugíneas; pedicelos pubescentes, de aproximadamente 0.2 a $0.3 \mathrm{~mm}$ de largo, articulados con las ramas de la inflorescencia.

Flores actinomorfas, de 4.5 a $5 \mathrm{~mm}$ de diámetro; cáliz con 5 sépalos fusionados cerca de la base, blanco verdosos, con una franja longitudinal verde en el centro, deltoides, de 0.9 a $1.1 \mathrm{~mm}$ de largo, pubescentes a lo largo de la vena media con tricomas blancos y rojos; corola de 5 pétalos libres, imbricados, blanco amarillentos, ovados, de 1.5 a $2 \mathrm{~mm}$ de largo y 1 a $1.5 \mathrm{~mm}$ de ancho, con 1 a 3 nervaduras longitudinales, verdes a negras, glabros.

Flores masculinas con disco de color rosa, anular, de $1.8 \mathrm{~mm}$ de diámetro, con diez lóbulos; estambres 10, que nacen en la parte inferior del disco, filamentos aplanados, más anchos en la base, de aproximadamente $1 \mathrm{~mm}$ de largo, anteras ovadas a ampliamente ovadas, de ca. $0.5 \mathrm{~mm}$ de largo y ca. $0.4 \mathrm{~mm}$ de ancho, dehiscencia longitudinal, con una glándula negra en el ápice del conectivo; pistilodios en el centro del disco, consistentes de 3 a 5 tubérculos cilíndricos, paralelos entre sí, de 0.2 a $0.3 \mathrm{~mm}$ de largo.

Flores femeninas con 10 estambres estériles de tamaño variable, de hasta $0.5 \mathrm{~mm}$ de largo y anteras de aproximadamente $0.2 \mathrm{~mm}$ de ancho; disco anular con 10 lóbulos, de hasta $2 \mathrm{~mm}$ de diámetro; gineceo de 5 carpelos unidos, color rosa, 
casi cilíndrico, cubierto de tricomas rojos glandulares, estilos 5, rojizos, separados entre sí y abiertos, casi horizontales, estigmas casi negros en seco.

Frutos drupáceos, cilíndrico-oblongos a obovoides, ligeramente asimétricos, de 1.1 a $1.2 \mathrm{~cm}$ de largo y 6 a $7 \mathrm{~mm}$ de ancho, con 3 a 4(5) cavidades desiguales, exocarpo delgado, pubescente, con puntos pelúcidos visibles con lupa (10x), mesocarpo ligeramente carnoso, muy delgado, en ocasiones algo fibroso, endocarpo óseo, asimétrico, con una proyección lateral cerca del ápice, con 3 a 4(5) opérculos desiguales, de contorno irregular, el más grande de aproximadamente $3 \mathrm{~mm}$ de diámetro, situado en la mitad superior, abriendo hacia el lóculo de mayor tamaño que contiene una semilla, los restantes más pequeños e irregulares, generalmente situados en la mitad inferior del ovario, abriendo hacia lóculos más pequeños sin semilla.

Semilla una, en el lóculo más grande, oblonga, ligeramente reniforme, testa de color café, con una mancha de color café oscuro a negro en forma de silla de montar, cotiledones blanco amarillentos, rectos, de 7 a $8 \mathrm{~mm}$ de largo y 2 a $3 \mathrm{~mm}$ de ancho, radícula de aproximadamente $2 \mathrm{~mm}$ de largo, formando un ángulo casi de $90^{\circ}$ con respecto al eje longitudinal de los cotiledones.

Esta planta es similar a Cyrtocarpa velutinifolia y $C$. caatingae, ambas especies de América del Sur, en lo que respecta a la consistencia ligeramente carnosa de las últimas ramas, hojas situadas hacia el ápice de las ramas que dejan la cicatriz muy marcada al caer, en la longitud y posición axilar de la inflorescencia en ramas terminales largas; difiere de $C$. velutinifolia en el número y tamaño de los folíolos, el tipo de pubescencia, tamaño del fruto y tipo de inflorescencia; de C. caatingae se distingue en que esta última tiene sólo 3 a 5 pares de folíolos, lanceolados a estrechamente elípticos y superficie de los folíolos serícea, pistilo glabro, ovarios y frutos uniloculares, mientras que C. kruseana tiene de (5)7 a 9(10) pares de folíolos elíptico lanceolados a elípticos de mayor tamaño, pubescencia no serícea, pistilo pubescente y ovarios y frutos con 3 a 4(5) lóculos.

Cyrtocarpa kruseana tiene corteza roja, lisa y exfoliante, a la manera de algunas especies del género Bursera, y sus últimas ramas son algo carnosas, arrugándose al secar, desarrolla sus inflorescencias sobre ramas terminales largas, tiene endocarpo óseo y los opérculos tienen forma variable y excepto el más grande, no están perfectamente delimitados, los opérculos más pequeños, en ocasiones, se observan como depresiones o cavidades que comunican hacia el interior de los lóculos. A su vez las especies mexicanas anteriormente conocidas, C. procera y C. edulis, tienen corteza gris, algo rugosa y no exfoliante, sus últimas ramas son de consistencia dura, no se arrugan al secar, generalmente producen inflorescencias en ramas laterales cortas, rara vez terminales, los frutos de C. edulis y C. procera tienen endocarpo duro, de superficie casi lisa, los opérculos están perfectamente 
delimitados, de manera que, al desprenderse, dejan una abertura de límites bien definidos.

Una diferencia más consiste en que Cyrtocarpa procera y $C$. edulis florecen entre marzo y mayo, mientras que $C$. kruseana lo realiza en julio y agosto, además de que habitan en lugares más secos que los sitios donde se desarrolla C. kruseana.

Nombre común y usos: Esta especie se conoce en Guerrero como "majahua" y "majahua de venado", su corteza interna se extrae en forma de tiras de hasta $2 \mathrm{~m}$ de largo y con ellas se realizan atados, se sujetan armazones de madera y se utilizan como correas para cargar los cestos donde se transportan diversos productos, uso que no se ha registrado en las otras especies mexicanas.

Distribución: Se ha colectado en Guerrero y se cuenta con ejemplares de Chiapas, Jalisco y Nayarit, por lo que es probable que se pueda presentar también en los estados de Oaxaca, Michoacán y Colima.

Altitud: 530 a $1000 \mathrm{~m}$. Florece en julio y agosto y se ha colectado con frutos en agosto y noviembre.

Tipo: México, Guerrero, municipio de Chilpancingo de los Bravo, $1 \mathrm{~km}$ al NE de Acahuizotla, antigua carretera México - Acapulco, altitud 1000 m, 25 de julio de 1982. A. Núñez 776 (holotipo: FCME; isotipo: NY).

Material adicional examinado: MÉXICO. Chiapas: Municipio Frontera Comalapa: 6-8 km east of Frontera Comalapa, along road to Ciudad Cuauhtémoc, altitud 1000 m, 23 de octubre de 1974, D. E. Breedlove 39045 (MEXU). Municipio Cintalapa: Near the microwave station of La Mina, $12 \mathrm{~km}$ south of Mexican Highway 190, near Rizo de Oro, altitud 1000 m, 16 de octubre de 1971, D. E. Breedlove 20649 (MEXU). Guerrero: Municipio de Juan R. Escudero: Alto Tepeguaje, altitud $680 \mathrm{~m}, 28$ de agosto de 1969, H. Kruse 2594 (MEXU). Alto Tepeguaje, altitud $680 \mathrm{~m}, 12$ de julio de 1970, H. Kruse 2801 (MEXU). Alto Tepeguaje, altitud $680 \mathrm{~m}, 4$ de agosto de 1970, H. Kruse 2847 (MEXU), 2848 (MEXU) y 2849 (MEXU). Alto Tepeguaje, altitud 680 m, 21 de noviembre de 1970, H. Kruse 2878 (MEXU). Aproximadamente a $1.5 \mathrm{~km}$ al SE del poblado Tepeguaje, laderas del cerro El Camal, altitud 530 m, 27 de agosto de 2003, R. M. Fonseca y E. Velázquez 3142 (FCME). Jalisco: Municipio La Huerta. Estación de Biología Chamela. Vereda Chachalaca metro 300, altitud $65 \mathrm{~m}, 4$ de julio de 2004, A. Pérez 3041 (MEXU), 3042 (MEXU). Municipio Tuxcacuesco. 5-6 km al OSO de Tuxcacuesco, 5-6 km al ENE de Zenzontla, cerro del Palacio, 16 de julio de 1991, F. J. Santana et al. 5157 (MEXU). Nayarit: Municipio Ahuacatlán: $\pm 13 \mathrm{~km}$ al 
NO de Ahuacatlán, altitud $\pm 1200 \mathrm{~m}, 19$ de julio de 1957, J. Rzedowski 9199 (MEXU). En El Ceboruco, derrame basáltico a $12 \mathrm{~km}$ al $\mathrm{N}$ de Ahuacatlán, carretera Guadalajara - Tepic, 8 de agosto de 1990, O. Téllez 12827 (MEXU).

Cyrtocarpa kruseana se distingue de las otras especies de Cyrtocarpa mediante la siguiente clave:

1. Ejes secundarios de las inflorescencias pseudoespigadas; hojas con 2 a 4 pares de folíolos de 4 a $9 \mathrm{~cm}$ de ancho. Plantas de América del Sur

C. velutinifolia (R. S. Cowan) J. D. Mitchell et Daly

1. Ejes secundarios de las inflorescencias paniculadas; hojas con 3 a 9(10) pares de folíolos de $3.5 \mathrm{~cm}$ de ancho o menos. Plantas de América del Sur o México.

2. Ovario 1 locular, glabro. Plantas de América del Sur

C. caatingae J. D. Mitchell et Daly

2. Ovario 2 a 5 locular, pubescente. Plantas de México.

3. Inflorescencias de 10 a 17 (20) cm de largo, agrupadas hacia el ápice de las ramas largas, densamente ferrugíneas; frutos de hasta $1.2 \mathrm{~cm}$ de largo; troncos con corteza roja, lisa, lustrosa, exfoliante; hojas con (5)7 a 9(10) pares de folíolos, con ápice agudo a acuminado, su pubescencia escasa en la madurez, restringida principalmente a las venas

C. kruseana Fonseca

3. Inflorescencias de $6 \mathrm{~cm}$ o menos de largo distribuidas a lo largo de las ramas generalmente cortas laterales, pubescencia no densamente ferrugínea; frutos de 1.5 a $2 \mathrm{~cm}$ de largo; troncos con corteza gris, opaca, algo rugosa, no exfoliante; hojas con 3 a 8 pares de folíolos con ápice redondeado a agudo, densamente pubescentes aún en la madurez.

4. Hojas con (2)3 a 5 pares de folíolos de 1 a $3 \mathrm{~cm}$ de largo; raquis cilíndrico, no alado. Plantas procedentes de Baja California Sur

C. edulis (Brandegee) Standl.

4. Hojas con (5)6 a 8 pares de folíolos de 4 a $7.6 \mathrm{~cm}$ de largo; raquis ligeramente alado. Plantas procedentes de Durango, Guerrero, Jalisco, México, Michoacán, Puebla, Oaxaca

C. procera Kunth

La especie se dedica al Sr. Hubert Kruse, importante colector en la región central de Guerrero principalmente en los municipios de Chilpancingo y Juan R. Escudero, quien colectó esta especie en diferentes épocas del año con el convencimiento de que era una nueva Anacardiaceae. Al ejemplar H. Kruse 2801 del mes de julio se anexa una nota que dice: "Me llamó la atención porque entre 
los demás árboles es el único defoliado todavía y apenas en botón" y de hecho en algunos ejemplares está escrito a mano "Anacardiaceae nueva".

\section{AGRADECIMIENTOS}

La autora agradece al Dr. Jerzy Rzedowski por la elaboración de la diagnosis en latín así como por la lectura del manuscrito original y las sugerencias para mejorarlo, al Biól. Ramiro Cruz, a la Biól. María del Rosario García Peña así como al M. en C. Mario Sousa del Herbario Nacional (MEXU) por facilitar la consulta del material colectado por Hubert Kruse, al M. en C. Ernesto Velázquez y al Sr. Carlos Ávila por su ayuda en el trabajo de campo.

\section{LITERATURA CITADA}

Barkley, F. A. 1957. A key to genera of the Anacardiaceae. Am. Midl. Nat. 28: 465-474. Engler, A. 1892. Anacardiaceae. In: Engler, A. \& K. Prantl (eds.). Die Naturlichen Pflanzenfamilien 3(5): 138-178.

Mitchell, J. D. \& D. C. Daly. 1991. Cyrtocarpa Kunth (Anacardiaceae) in South America. Ann. Missouri Bot. Gard. 78: 184-189.

Wannan B. S. \& C. J. Quinn. 1991. Floral structure and evolution in the Anacardiaceae. Jour. Linn. Soc. London, Bot. 107: 394-385. 